\title{
5G technologies boosting efficient mobile learning
}

\author{
Helen C. Leligou ${ }^{1, *}$, Emmnouil Zacharioudakis ${ }^{2}$, Louisa Bouta $^{2}$, Evangelos Niokos $^{2}$ \\ ${ }^{1}$ Technological Education Institute of Sterea Ellada, Electrical Eng. Dept. Psahna Evias, 34400, Greece, leligou@gmail.com \\ ${ }^{2}$ OTE Academy, Pelika \& Spartis 1, Maroussi, 151 22, Athens, \{manos.otea@gmail.com, lbouta, vniokos\}@oteacademy.gr
}

\begin{abstract}
The needs for education, learning and training proliferate primarily due to the facts that economy becomes more and more knowledge based (mandating continuous lifelong learning) and people migrate among countries, which introduces the need for learning other languages, for training on different skills and learning about the new cultural and societal framework. Given that in parallel, time schedules continuously become tighter, learning through mobile devices continuously gains in popularity as it allows for learning anytime, anywhere. To increase the learning efficiency, personalisation (in terms of selecting the learning content, type and presentation) and adaptation of the learning experience in real time based on the experienced affect state are key instruments. All these user requirements challenge the current network architectures and technologies. In this paper, we investigate the requirements implied by efficient mobile learning scenarios and we explore how $5 G$ technologies currently under design/testing/validation and standardisation meet these requirements.
\end{abstract}

\section{Introduction}

Lifelong learning becomes more and more important for the EU economy in its way towards an innovation-based economy where disruptive out-of-the-box thinking and fast adoption of new (including digital) technologies plays an ever increasing role. In parallel, EU society is becoming more and more multi-national as large flows of immigrants arrive in diverse countries making it hard to design training programs and even harder (and very expensive) to realise them. At the same time, in the socalled new EU member states (like Hungary, Bulgaria, Romania, Slovakia) high unemployment coexists with skills shortages; business and industry criticise quality and relevance of current training programs; low image and attractiveness of Vocational Educational Training (VET) has impeded its penetration even though higherlevel qualifications bring higher returns. Hence, there is a clear market need for reinforcing vocational education and training, providing incentives to better match skills and jobs, offering attractive VET programmes to engage both young and older people, even with poor prior education, low aspirations and motivation and/or from deprived backgrounds.

The exploitation of state-of-the-art information technologies, and more specifically those relevant to mobile devices, can significantly assist in increasing the effectiveness of all types of education while lowering its cost, meeting the needs of both learners and tutors. The learners want to be able to easily access learning material at anytime, anywhere so that they productively use all their available time. At the same time, attractive learning material employing gamification concepts strengthen user engagement and improve the learning experience. Finally, social interactions have become an indispensable flavor of all our daily activities and in education contexts they could be exploited to cultivate competition.

From the tutor perspective, being able to reach the learner at any time contributes to the faster achievement of educational goals. Additionally, being able to easily develop customized (or even personalized) learning curriculum and material, reusing already available ones, significantly accelerates their work. With the assessment of learner performance being another time consuming task, its easy (automated) assessment exploiting Information Technologies becomes important; even more so, the automated assessment of the learner's behaviour (exploiting sensing devices and data fusion) can drive further personalization of the learning experience.

Towards meeting these needs, the ever increasing penetration of mobile devices (smart phones and tablets) throughout the world can be leveraged. Learners of all ages are familiar with mobile apps including games and cloud-based applications. Mobile apps enable the seamless communication among the learners, and between learner and tutor. Furthermore, the large cloud infrastructures can be used for big (learning) data storage and complex processing. State-of-the-art smartphones are equipped with sensors enabling a) the creation of more attractive learning materials (e.g. games using gyroscopes and other sensors of the smart devices) and b) the assessment of user engagement. Emotion recognition based on facial expressions analysis is possible [1]. Distinguishing boredom in real time allows

\footnotetext{
Corresponding author: leligou@gmail.com
} 
for triggering experience adaptation to personal needs in real-time.

The development of smart mobile applications that use the sensors available on these devices to detect and personalise the learning experience and communicate with a cloud infrastructure to perform heavier storage and processing tasks is nowadays feasible. Such smart educational solutions are expected to increase the effectiveness of education [2] at all levels and reduce the relevant cost through the sharing of learning resources.

These trends are not at all EU specific and this is proven by the global statistics and forecasts for the elearning market: PRNewswire stated (in March 2017) that "the Global E-Learning Market is poised to grow at a CAGR of around $7.2 \%$ over the next decade to reach approximately $\$ 325$ billion by $2025 "$ with mobile learning market growing fast (to reach $\$ 12.2$ billion in 2017 [3]). Education is the third most popular industry in the app development market which is $9.44 \%$ of all active apps in the Apple App Store. Organizations use more and more mobile devices in their training programs to save time from their employees' lives, to have more and more answers in their fingerprints through their smartphones, to enable Bring Your Own Device (BYOD) paradigm in training allowing them to benefit of any timeslot in their busy schedule. Gartner predicts that $45 \%$ of businesses will have a BYOD policy by 2020 (Gartner forecasts). This is also becoming true for children education (k-12 training) that can enjoy playful experiences at home strengthening their education. MarketsandMarkets forecasts the Global mobile learning market to grow from $\$ 7.98$ billion in 2015 to $\$ 37.60$ billion by 2020, at a Compound Annual Growth Rate (CAGR) of $36.3 \%$. Additionally, $70 \%$ of learners felt more motivated when training on a mobile device [4]. Mobile e-learners typically study for 40 minutes longer than students using a desktop or tablet [5]. Smartphone learners complete course material $45 \%$ faster than those using a computer. Especially, younger people seem to prefer robot-based learning or combination of laptop/mobile/robot learning sessions, with interactive whiteboards also being widely used to offer an enhanced learning experience.

Mobile learning owes its attractiveness to three key features, all tightly coupled with the mobile network performance and features. Firstly, it enables self-directed anytime, anywhere learning; secondly, convenience during the busy day supporting personalisation of training schedules and thirdly but most importantly, it leads to better outcomes: increased retention, reduced training times, and boosts productivity than more traditional training models. Serious games and real-time adaptation of learning experiences are the cornerstones of this last feature.

In this paper, we identify the key features of $5 \mathrm{G}$ technologies that open the way for improved mobile learning solutions to the benefit of both the society and the market. The rest of the paper is organised as follows: section 2 presents typical architectures of mobile learning solutions specifying requirements for improved learning solutions and technical points where improvements are possible; section 3 presents key advantages of $5 \mathrm{G}$ technology while section 4 attempts to guide engineers in exploiting $5 \mathrm{G}$ innovation for delivering mobile learning solutions with advanced efficiency.

\section{Mobile Learning architectures}

The fast growing mobile learning sector includes many different solutions and applications ranging from mobile apps downloaded and executed in mobile devices (smartphones and tablets) running any operating system, to fully connected experiences where multiple learners interact in real time (play training games) through different and diverse devices-obviously mediated by a cloud infrastructure. In between the two boundaries, solutions where learning materials and/or schedules and/or other learning relevant information is kept in some cloud infrastructure accessed by the learner to initiate a new learning session can be found. While the first type of mobile learning does not really challenge the network operation, internet-enabled or internet-based solutions can impose really demanding requirements on the network performance.

Different architectures are surveyed in [6] where they are finally evaluated and compared with respect to a set of factors that include: 1) device mobile support, 2) heterogeneity support, 3) wireless communication technology, 4) adaptability, 5) traceability and control, 6) learning as a collaborative process and 7) learning integrated with formal or realising informal learning. The reason for selecting these factors is that they are tightly coupled with the user requirements for contemporary mobile learning scenarios and foremost learning process personalisation.

\subsection{Learning process personalisation}

The personalisation of the learning process is crucial for high learning performance. The performance depends on what the user experiences which in turn is reflected in his affect state. Personalization can involve allowing learners to customize the learning experience by selecting a visual theme or accommodating individual comfort and accessibility or by enabling learners to control volume and screen text font sizes. Personalization can be taken further by capturing a learner's name during registration and using it throughout the course or by streaming specific content to an individual. To increase learning efficiency, the learning infrastructure has to provide the appropriate content for every person at each time, in the appropriate for every person presentation form and level of difficulty. To decide the "appropriateness" of content and presentation, pedagogical models are used, which take into account the person's skills and more interestingly the person's emotional state. To decide the affect state, the sensors of the device in use have to be exploited to collect readings to feed affect-state detection modules so as to decide on keeping or changing certain parameters of the learning experience, such as difficulty, or even changing the learning material itself to boost the 
learner's engagement. With regards to skill acquaintance towards personalising the learning experience, the performance of the learner has to be continuously traced. The intelligence of adapting the learning experience to the learner's skills and emotional state needs also to take into account past behaviours and process evolutions and thus cannot be implemented (locally) in the mobile devices. In this case placing the personalisation decision module to some cloud infrastructure, introduces the requirement for exchanging important volumes of data between the mobile devices and the cloud.

The volume of the data communicated between the mobile device and the intelligent components residing in the cloud as well as the relevant latency requirements, depend on the type of sensors used to extract emotion information as well as on the type of learning material and learner performance metrics of interest. If camera readings are used for emotion detection, the volume of data is quite big while emotion detection is still possible (at coarser levels) using gyroscope and accelerometer readings. [7] Up to now, streaming the mobile device's camera reading to the cloud is considered an impractical solution which however, would result in higher accuracy in emotion detection. With respect to the learner's performance, pedagogists and trainers are responsible for identifying key moments, where the interactions between the learner and the learning material has to be captured so as to drive learning performance personalisation. For example, if the learning material is a video demonstrating the assembly of a certain device, the "performance" is expressed just as "video watched" while the case is significantly different if the learning material is an interactive game where the two trainees are immerged in the same critical situation and the aim is to assess their reactions towards crisis management. To respond to individual needs, pre-testing is an effective method based on existing competencies. The pre-test assessment can consist of a series of questions linked to course topics and allow learners to skip topics linked to correctly answered questions.

\subsection{Collaboration and gaming}

Collaboration during learning was initially considered as one of the disadvantages of distant learning. However, collaboration either for assisting one-another or even for solving the same problem can be considered today a mainstream app. On the other side, gamification is introducing competition and has been shown to increase engagement as it usually offers a playful experience. Both real-time collaboration and gamified learning content impose strict requirements on the network supporting the communication between the different learners in terms of latency. The difference of this situation from any other multi-player gaming app from the network perspective is that apart from information regarding the players' interactions, information about the learners' performance needs to be traced and communicated to/through the cloud as well as the data from the devices sensors to enable affect detection and learning process adaptation in real-life.
The situation becomes even more challenging when the collaborating or playing tenants use diverse devices which can either be any mix among mobile (smartphones and tablets), fixed (like whiteboards) and robots. In this case, the information needs to be communicated in a standardised manner (e.g. xAPI statements) to enable appropriate processing in the cloud towards tailoring the presentation of the interaction to the learner and device combination in place each time.

\section{Critical Requirements}

-Personalised learning involving mobile devices imposes the following requirement on the network: Enhanced mobility support to enable learners exercise everywhere and anytime, even when in the bus or train, so as to exploit all available timeslots in their busy schedules.

-High capacity/low latency to enable data from the sensing elements of the mobile devices to reach cloud infrastructures where "intelligence" allowing personalisation and real-time adaptation based on affect detection resides.

-High capacity/low latency to enable performance relevant data reach the cloud where the learning experience is decided andcontrolled. The modern learner wants to know how information relates to and benefits them directly. Learnercentered eLearning provides opportunities for learners to relate content to their role or personal situation through thought-provoking self-reflective learning checks. These are particularly powerful when interspersed within a scenario at pivotal moments to provoke learners to think how they would handle the situation.

-High security and privacy support: Privacy and security become more and more important in our lives, challenging the networks as they become more and more heterogeneous and as they rely on decoupling physical from logical topologies.

-Quality of Service: The data exchanged between the mobile device and the cloud infrastructure can be distinguished in data relevant to the execution of the learning material (game or other), data relevant to affect detection (generated by the mobile device's sensors) and data relevant to the performance of the learning allowing for self-reflection as well as for experience personalisation. These three types of data need differentiated treatment if resources are to be efficiently used over any network that connects the mobile device with its peers.

-Long battery lifetime: Having the mobile devices executing learning materials (e.g. games) with almost all sensors operating in parallel to enable affect detection and learning experience personalisation, prolonging the lifetime of the battery becomes mandatory to accomplish e.g. 
an one-hour experience while on move (e.g. at train).

These requirements should be considered on top of the continuous connectivity and network reliability and availability which are also imposed by traditional learning scenarios.

\section{5G-features relevant to the mobile learning use case}

5G will provide an order of magnitude improvement in performance in the areas of more capacity, lower latency, more mobility, more accuracy of terminal location, increased reliability and availability. 5G will allow the connection of many more devices simultaneously and to improve the terminal battery capacity life. Lastly, 5G will help European citizens to manage their personal data, tune their exposure over the Internet and protect their privacy.

5G offers more capacity: 5G architectures envision capacity in the range of $1-10 \mathrm{Gbps}$ to the wireless edge network part with multiple $10 \mathrm{Gbps}$ for the access part and even higher for the metro part. What is even more interesting and important is not the capacity itself but the capability to manage the capacity enjoyed by each service and the capability to isolate traffic in each socalled network slice. Network slicing, in $5 \mathrm{G}$ vision, is also an important part of the overall 5G architecture that addresses the deployment of multiple logical networks as independent business operations on a common physical infrastructure. One of the goals would be to provide network slices flexibly meeting the wide range of use cases that the 2020 timeframe will demand [8]. To this end, a "5G slice" could be composed of a collection of $5 \mathrm{G}$ network functions (NF) and specific radio access technology (RAT) settings that are combined together for a specific use case and/or business model. In that context, network slices must fulfil a set of requirements such as the need for sharing and efficiently reusing resources (including radio spectrum, infrastructure, and transport network); differentiation of traffic per slice; visibility of slices; protection mechanisms among slices (a.k.a. slice isolation); and support for slice-specific management. Isolating traffic of different logical networks allows for achieving lower latency for service that require it as is the case for the interactive mobile learning scenario in combination with the bulk of data that need to be exchanged not only for the execution of the learning material but equally importantly for the communication of sensed and performance-relevant data.

Transport slices should also support seamless VNF mobility within the slice. 5G Converged Data Plane functions are distributed to the edges of a common core network, resulting in creating a distributed flat network. The control plane functions, which are responsible for mobility management, QoS control, etc., direct the user traffic to be served agnostically to the access networks to which it is attached.

Network functions in $5 \mathrm{G}$ will be more strongly decoupled from physical architecture than in legacy systems Traditionally, mobile network functions are implicitly grouped into network entities via specification of their interconnections, where each entity is responsible for a pre-defined set of functions. A balance between flexibility and complexity needs to be taken into account. In 5G systems, network functions are to be designed to allow a maximally flexible instantiation or even dynamic (re)allocation of functions (i.e. logical entities) to physical entities, enabled by the principle such as a) avoidance of strict timing relations between network functions and protocol stack layers, and design of network functions and b) design of network functions such that these are either able to adapt to the physical architecture in which they are used or are replaceable by alternate network functions specifically optimized for these non-ideal environments. Furthermore, the support of the design, implementation, deployment, management and maintenance of network functions by software programming, exploiting characteristics of software such as flexibility and rapidity of design, development and deployment throughout the lifecycle of network functions etc. can be seen as replacing a "network of entities", as in legacy systems, by a "network of (virtual) functions."

These network functions certainly include mobility, traffic control, authentication, authorisation, accounting and security as well as Intrusion Prevention and Intrusion Detection. Mobility management function design and selection may be service-specific, to allow for a higher degree of customization, e.g., network-slicespecific or radio-access-specific mobility management. This perfectly suits learning sector requirements for enhanced mobility, as a significant part of users exploit the time they spend traveling/commuting between office and home to learn, imposing stringent requirements on the network with respect to mobility management.

With respect to security aspects, the decoupling of logical functionality from its physical realization has always required dedicated security mechanisms. The fact that $5 \mathrm{G}$ networks and functions will, to an even higher degree, be provided as logical/virtualized concepts, emphasizes both the required scope and the criticality of security. Most of the necessary security functions in current networks are flexible in the sense that they can "move along" with the motion of the functionality that they protect. But this does not mean that security is agnostic of the physical realization of the logical architecture. $5 \mathrm{G}$ vision is to offer enhanced security and flexible AAA over heterogeneous networks. Servicespecific access control and accounting/charging policy functionality and placement will be supported as well as service specific security (e.g. a slice with no encryption and/or with added data integrity). 5G aims at designing a security architecture that is flexible and extensible, allowing e.g. functional end-points to be re-allocated due to mobility or traffic optimization. Nevertheless, a complete independence of the physical architecture cannot be obtained. It plans for service-specific access control to allow or block access to deployed services and service-specific monitoring to detect suspicious traffic. Configuration of the Intrusion Prevention and Intrusion Detection VNFs will depend on the type of services that require protection and on the security policies set. 
Decentralised security and privacy are of utmost importance to the $5 \mathrm{G}$ vision.

Other key characteristics of $5 \mathrm{G}$ architectures to be exploited in mobile learning scenarios are the increased reliability and availability as well as the improved terminal battery capacity. Reliability and availability rely on the multi-connectivity technology implemented in 5G in combination with the network function virtualisation which will promptly decide on the physical network reconfiguration. On the other hand, terminal battery capacity improvements primarily depend on the sleep time and patterns that can be enforced on a service specific manner.

\section{How 5 G meets learning requirements}

The growing demand for learning through mobile devices in diverse environments moving (as in train) or not (at home) presents challenges to cellular networks. The imposed requirements are already addressed by use cases from other vertical industrial sectors on which currently ongoing projects focus on. In this framework, they have narrowed down the generic goals of the $5 \mathrm{G}$ PPP group vision to concrete target values for Key Performance Indicators. These are listed in the following table.

Starting from the increased user data rates, the means to achieve this goal are to use more frequency spectrum and more dynamic spectrum access. Towards reducing significantly the end-to-end latency, the primary methods are to use more efficient network architectures, more efficient air interface designs, signaling and procedures, better QoS differentiation, and direct device to device communication, where possible. With respect to network architectures, low-latency network architectures are concerned with shortening the distances and number of hops between the user and the content; for example, by distributing some network functions that are centralized today. On the other hand, the air interface may adapt the frame structures in order to reduce the Transmission Time Interval (TTI) and/or Hybrid Automatic Repeat reQuest (HARQ) round trip times. While this can efficiently be implemented at the higher frequencies where more bandwidth is available, this can become very inefficient for wide area coverage. Signaling and procedures can be thinned out, by simplifying their components. Turning our attention to better QoS differentiation, as the low-latency transmission usually comes with inefficient resource utilisation, it should only be applied with the services that require such low latencies.

Starting from the requirement to support fast service deployment, activation and further management, this is in essence the efficient mapping of service functions onto a virtualized substrate, possibly belonging to multiple infrastructure operators. For the service deployment time, in other words, the duration required for setting up end-to-end logical network slices characterized by respective network level guarantees (such as bandwidth guarantees, End-to-End (E2E) latency, reliability), stringent target values are imposed by media and automotive use cases.

With respect to the battery longer lifetime, the primary methods to address its prolongation are to improve air interface, procedures and signaling, and to reduce the distance between the Massive Machine Communications devices and the access node. Techniques to improve the air interface include the use of sleep modes, energy-efficient modulation, coding and multiple access schemes, for example, use of constrained modulation techniques, very robust coding and/or spreading allowing very low transmission powers, and simplified procedures such as those introduced for the previous goal [9]. As far as security is concerned, tailored security at the service and device level is envisioned in $5 \mathrm{G}$ vision. $5 \mathrm{G}$ might consider dynamic control and data plane support for different security system instantiations to be able to provide differentiated security services on request.

Table $15 \mathrm{G}$ technology responses to Use Case requirements

Mobile Learning Use Case Requirements

Enhanced mobility support to enable learners exercise everywhere and anytime

High capacity/low latency to support data handling differentiation for sensed data and performance related data; these requirements are tightly bound to Quality of Service differentiation capabilities

High security and privacy support

Long battery lifetime

Network reliability and availability
5G Architecture Response

Fast Service deployment

Support Velocity $>50 \mathrm{~km} / \mathrm{h}$ (currently targeted in automotive use cases projects)

5G target : 1000 times higher mobile data volume per area

The user data rates considered in the $5 \mathrm{G}$ use case go beyond $\geq 1$ Gbps while for latency, three different levels are distinguished with the low one ranging from $1-10 \mathrm{~ms}$ Furthermore, multiple traffic types are supported between them bursty, periodic and event driven.

Increasing importance in the highly heterogeneous, rapidly changing, network architecture

10 times longer battery life based on sleep time and patterns that can be enforced on a service specific manner.

Target reliability ranges from low $(<95 \%)$ to high $(>99 \%)$

Target availability also ranging from low $(<95 \%)$ to high $(>99 \%)$ 
The dynamic composition of the $5 \mathrm{G}$ infrastructure needs security guarantees within the system: beyond the mutual authentication and secure communication channel establishment, and despite the decoupling between logical and physical network topologies, it is necessary to delve into topics of infrastructure/system integrity and operational security assurance. As the system dynamics are key to achieve the agility of stakeholders (as recent Network Function Virtualisation and Software Defined Networking initiatives suggest), then the survivability must be increasingly understood as the major operational security model.

\section{Conclusions}

As the realm of mobile learning augments, it is important to identify technologies that best meet the user requirements. Supporting personalisation and real-time adaptation to the temporal user needs leads to enhanced user satisfaction, reduces significantly the achievement of the learning goals to the benefit of the individual, the society and the economy. Our investigation shows that 5G technologies are anticipated to significantly boost mobile learning as it efficiently satisfies high priority user requirements providing the tutors/trainers with richer means to define efficient learning scenarios. Among the most important $5 \mathrm{G}$ features, high data rates enable communication of performance and affect-state relevant data which fuel learning experience personalisation and adaptation; high battery lifetime offers the user the opportunity to learn throughout long journeys; quality of service differentiation and high network availability and reliability contribute in enhancing the learning experience through a variety of learning content types from simple videos and files to interactive possibly collaborative learning games. 5G provides the engineers with abundant tools to implement an unprecedented number of novel learning solutions meeting learners' and trainers' requirements making learning more enjoyable and efficient than ever.

\section{Acknowledgment}

This work has been co-financed by the EU H2020 MaTHiSiS project.

\section{References}

1. ICT-317669 METIS project, Scenarios, requirements and KPIs for $5 G$ mobile and wireless system (Deliverable D1.1, May 2013), available at: https://www.metis2020.com/documents/deliverables

2. L. Duan, D. Xu, I. W. Tsang, Learning with Augmented Features for Heterogeneous Domain Adaptation, Proceedings of the 29th International Conference on Machine Learning (Edinburgh, Scotland, UK, 2012)

3. https://elearningindustry.com/surprising-mobilelearning-statistics-elearning-professionals-know
4. Lynda.com, The benefits of mobile learning, white paper, (2014), available at: http://cdn.lynda.com/cms/asset/text/lyndacomwhitepaper-mobile-learning-benefits20151245042329.pdf.

5. http://mnalearning.com/resource view.php?id $=70$

6. S. Baccari, F. Mendes, C. Nicolle, F. Soualah-Alila, N. Mahmoud, Comparative Study of the Mobile Learning Architectures, e-LEOT 2nd EAI International Conference on e-Learning eEducation and Online Training, ( Novedrate, Italy,2015)

7. A. Færøvig Olsen, Detecting Human Emotions Using Smartphone Accelerometer Data, (Master Thesis, 2016)

8. 5G PPP Architecture Working Group, View on $5 G$ Architecture (2016), available at: https://5gppp.eu/wp-content/uploads/2014/02/5G-PPP-5GArchitecture-WP-July-2016.pdf

9. J. F. Monserrat, G. Mange, V. Braun, H. Tullberg, G. Zimmermann, Ö. Bulakci, METIS research advances towards the $5 G$ and wireless system definition (EURASIP, 2015)

10. M. Maternia, S. E. El Ayoubi, 5G PPP use cases and performance evaluation models (2016), available at: $\underline{\text { https://5g-ppp.eu/wp- }}$ content/uploads/2014/02/5G-PPP-use-cases-andperformance-evaluation-modeling v1.0.pdf

11. M. Suk, B. Prabhakaran, Real-time Mobile Facial Expression Recognition System - A Case Study, IEEE Conference on Computer Vision and Pattern Recognition Workshops (2014)

12. Conference on e-Learning e-Education and Online Training ( Novedrate, Italy, 2015)

13. A. Mecke, I. Lee, J.R. Baker jr., M.M. Banaszak Holl, B.G. Orr, Eur. Phys. J. E 14, 7 (2004)

14. CEDEFOP, Spotlight on VET in Hungary(2014)

15. CEDEFOP, Spotlight of VET Romania (2013)

\footnotetext{
Corresponding author: leligou@gmail.com
} 